\title{
Design and Implementation of Intelligent Water Dam Monitoring System using Fuzzy Logic
}

\author{
Sawsan A. Arif and Rabah N. Farhan \\ Computers and Information Technology College, \\ Renewable Energy Research Center, University of Anbar, Anbar, Iraq \\ azalsawsan@yahoo.com
}

\begin{abstract}
Iraq has constructed a number of dams for generating hydroelectric power and for irrigation. As it is a critical civil infrastructure for the development of the country, an automated method to keep track of dam safety achieved in this study. This system works by obtaining useful information about the behaviors of the dams, predict the future values and detect abnormalities (irregularities) as early as possible to enable a timely response as fast as possible. In this study, a wireless sensor network application for dam safety monitoring task where investigated. The sensor network encompasses heterogeneous types of sensors distributed in different portion of a dam to gather data about the integrity of the dam. The sensor nodes are organized and spread across the body of dam and has the central node. Fuzzy inference system used to adjust and maintain the time and amount of dam gate opening and closing depending on the state of the dam. The system also supplied with SMS communication for remote communication across the country. The system allows real-time connection and processing of sensor data that presenting to the user through an appropriate interface written in $\mathrm{CH}$. The overall performance of the system tested on dam benchmark. The evaluation of the system shows that it adapt well with the vibration and sudden increasing level of water.
\end{abstract}

Key words: Fuzzy logic, dam monitoring, GSM network, vibration, ultrasonic sensor, Arduino Uno, servo motor

\section{INTRODUCTION}

The promising field of wireless sensor networks involves sensing, computation and communication into a single tiny device. This open the door into new applications (Sohraby et al., 2007). Wireless sensors have been developed for different applications such as military, monitoring, medical health monitoring, structural physical condition monitoring, etc. (Shukla and Pandy, 2014). Dam is a critical civil infrastructure for many nations. For instance, Iraq has constructed a number of dams (Fig. 1). As it is an important general infrastructure for the development of the country, an computerized method to keep track of dam safety by gathering helpful information about the behaviors of the dams, predict the future values and detect abnormalities (irregularities) as early as possible to enable a timely response is thus, of utmost importance. Dam Safety Monitoring (DSM) works by examining the health of the existing dam by a team of persons who are qualified to evaluate a particular structure (Schurer et al., 2002).

Dam failures are most likely to occur for one of the following ways (Kavanagh, 2011). The first cause is due to water flooding over the dam which accounts for

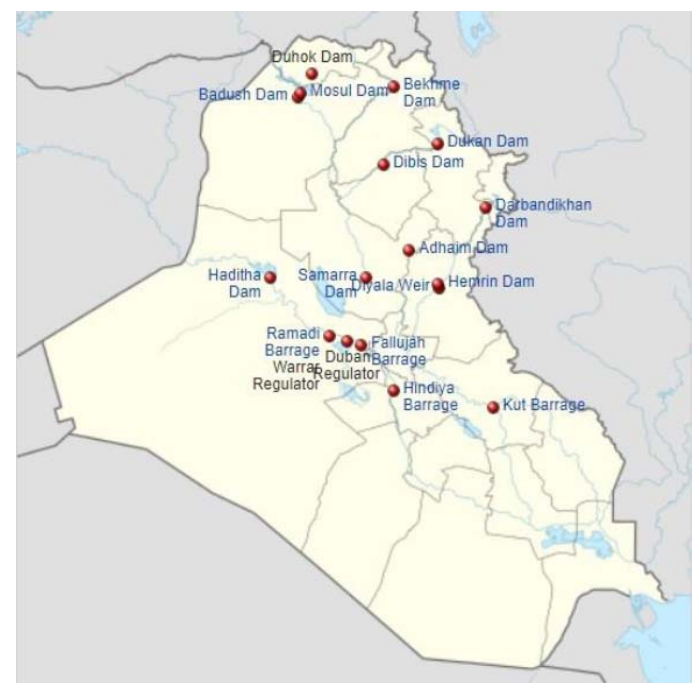

Fig. 1: Water dams in Iraq

approximately $34 \%$ of all dam failures. The second reason is furious caused by the vibrations of dam that share for $30 \%$ of all dam failures. The third reason for dam failure caused by internal erosion.

Corresponding Author: Sawsan A. Arif, Computers and Information Technology College, University of Anbar, Anbar, Iraq, azalsawsan@yahoo.com 


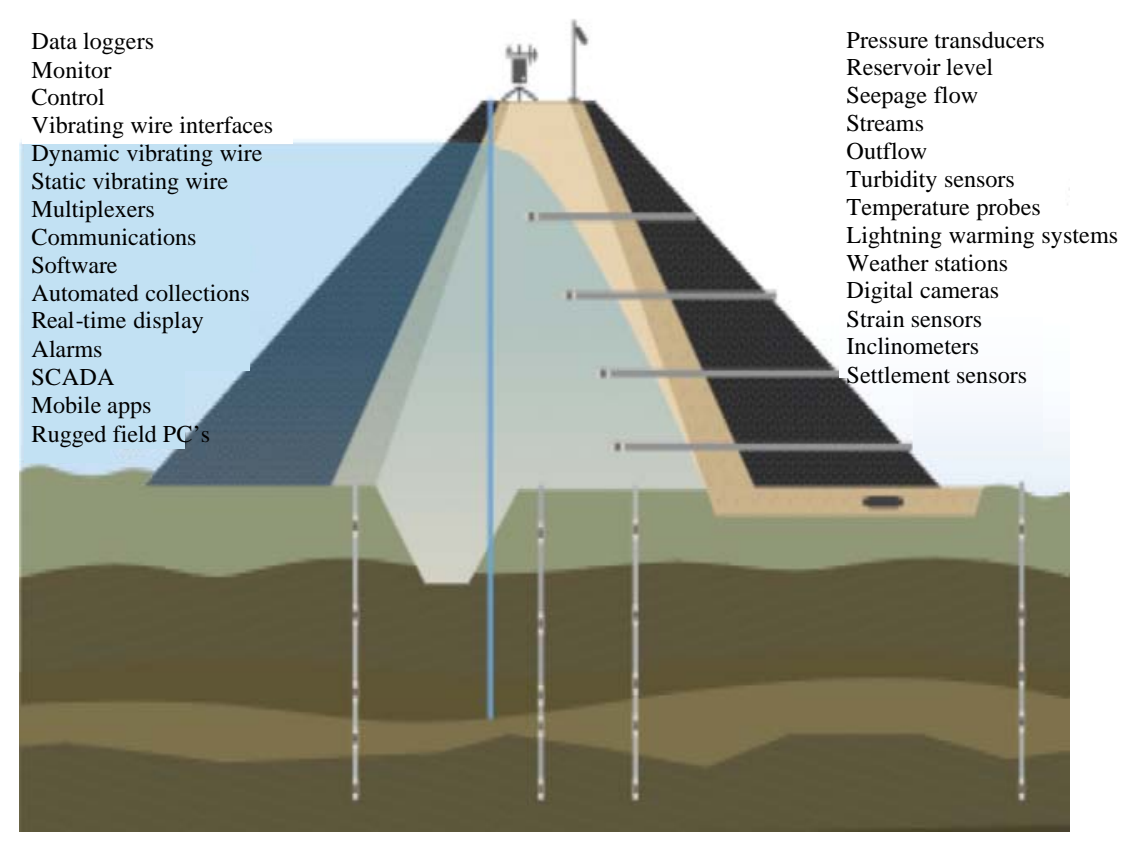

Fig. 2: DMS structural organization (data acquisition system for dams)

Iraq has a range of water dams spread over the Tigris and Euphrates Rivers as shown in Fig. 1. The government made great efforts to maintain these dams which severely damaged by wars and by earthquakes that occur from time to time. There is an urgent need for the development of a comprehensive monitoring system that monitors the dams and obtains data about the condition of the dam and the level of water and the associated vibrations in the body of the dam periodically (Schurer et al., 2002).

Dam monitoring system: Dam Monitoring System (DMS) is made to be as distinctive and robust as possible due to deep impact of risks that may be caused by failures in DMS (Fig. 2). They assess water pressure, flow and turbidity, dislocation, strain, load, vibration and many other parameters (Fig. 3). DMS supplied with a full range of network capabilities, real-time user interfaces, alarm capabilities and compatibility tools with other systems (Talgote et al., 2015).

Wireless Sensor Network (WSN): Wireless networking is an emerging technology that makes the users to access data and services electronically, irrespective of their geographic location. The wireless network is a data communication system which uses electromagnetic waves to transmit and receive information via. air as medium from one place to another place. Essentially, wireless networks are classified into two types (Sazonov et al., 2004; Miao et al., 2012): infrastructure-based networks and infrastructure-less (ad-hoc). The ad-hoc networks compromise low cost wireless technologies, such as Bluetooth, Zigbee/IEEE 802.15.4, etc. (Manes et al., 2007).

They were very suitable in controlling and monitoring of water dams. Zigbee WSN which is able to form large networks can be used to maintain the formation of WSN to cover the dam body. Theoretically, Zigbee network structure can connect over 65,000 nodes. In addition, Zigbee network provide low power consumption via. multi-hop technology and has the option to self-organize the whole sensor network (Pan and Yu-Chee, 2006). WSN play major roles in DMS because they were very effective in achieving the goals set for them and their efficiency in obtaining data from the sensors distributed on the body of the dam to the central station (Koubaa et al., 2006; Wagh and Rao, 2014).

Fuzzy logic control: Fuzzy logic control is a system implemented on high-performance query processing, including database queries and transactions that make traditional world deal with fixed deterministic, precise values. Information systems often based on very large data collections, mostly stored in relational databases (Imran et al., 2016). Fuzzy logic, unlike the statistical data mining techniques such as cluster or regression analysis, enables the use of non-numerical values and introduces the notion of linguistic variables. Using linguistic terms and variables will result in a more human oriented 


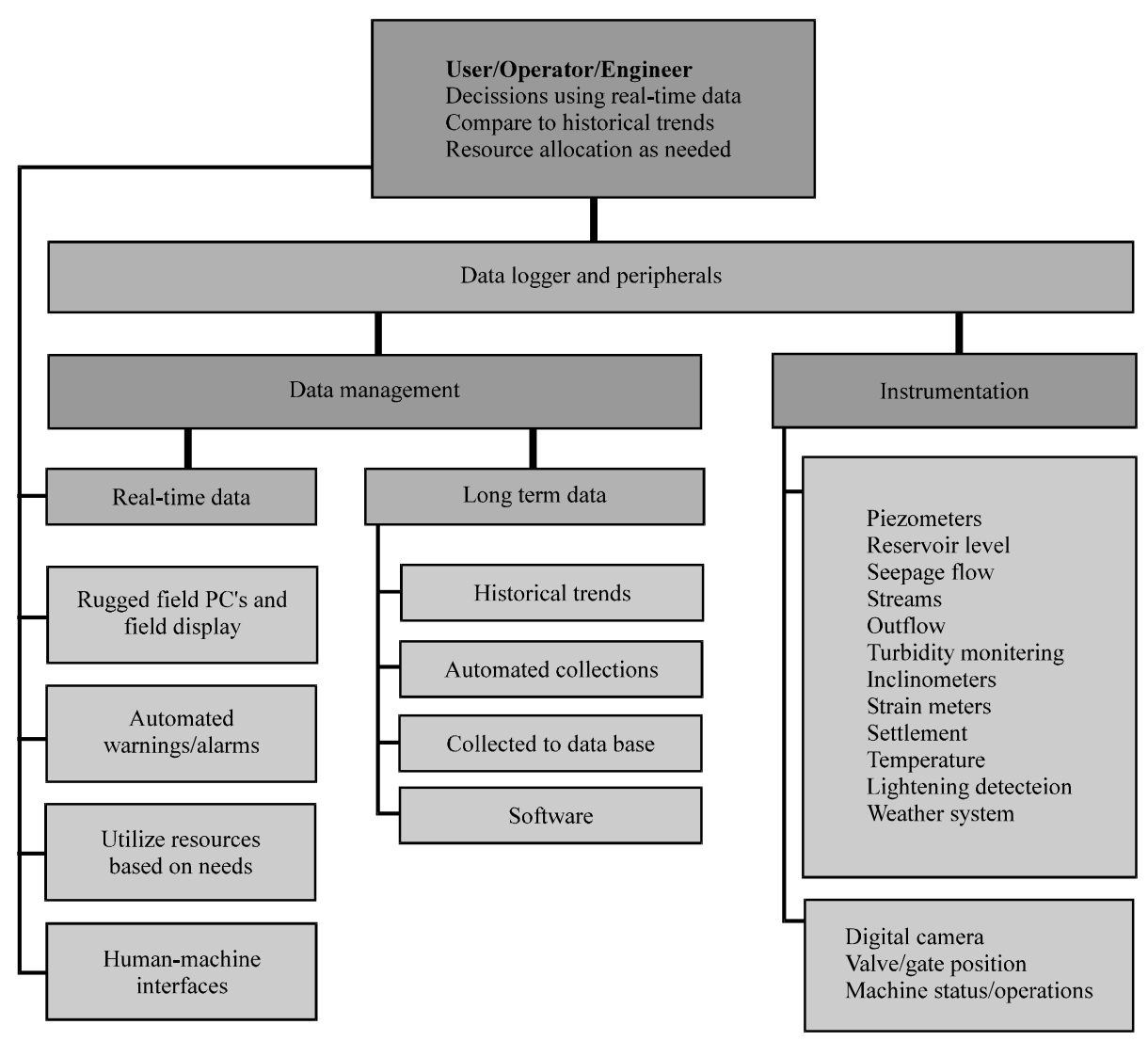

Fig. 3: DMS functional organization

querying process. The fuzzy classification achieved by extending the relational database schema with a context model. Fuzzy inference process formulates the mapping of a given input to an output using fuzzy logic. The mapping provides a basis from which decisions were made. The process of fuzzy inference includes membership functions, fuzzy logic operators, if then rules and knowledge base. The fuzzy based system decides the gate opening limit value to avoid the damage caused by water flooding (Nanaware et al., 2012).

Literature review: Many researchers have contributed and addressed the problem of DMS. Sazonov et al. (2004) presents the concept of a sensor network for fully autonomous structural health monitoring addresses the critical issues of integrating a sensor network with a vibration-based method (Sazonov et al., 2004). Hu and Liu (2011) proposes solutions for tailings dam safety monitoring, designs a software structure which includes visualization, automation and intelligent control. He discusses some critical technologies in this system. The security monitoring system developed which offer a reference for researching and developing tailings dam security monitoring system (Hu and Liu, 2011). Nanaware et al. (2012) attempting to develop a Fuzzy Inference System (FIS) using the fuzzy tool in MATLAB. The model study different types of Membership Function (MF) and defuzzification. The experience of the operator and available documentation utilized to design FIS for gate limiter. This study presents the use of fuzzy logic in Hydro Power Plant (HPP) to decide a maximum opening value for the gate (Nanaware et al., 2012).

Miao et al. (2012) a Wireless Dam Sensor Network (WDSN) presented in this study. The WDSN consists of smart nodes and a sink node. The power saving strategies at various levels, from the network cluster architecture, to Zigbee communication protocol, the sensor nodes architecture and the dynamic response mechanism explained. The experimental results demonstrate the reliability of transmission (Miao et al., 2012).

Urdiain et al. (2012) presents an Arduino-based wireless sensor network to monitor parking lots using a non-standard low-power energy-balanced system. The event-driven routing protocol follows the hierarchical clustering philosophy. Energy preserved by minimizing the number of transmissions needed to forward 
information to the base station. The smart sensor platform is built using the popular Arduino development platform, Sharp IR distance sensors and $\mathrm{nRF} 24$ low-power radio modules (Urdiain et al., 2012).

Seid (2013) develops a wireless sensor network application for dam safety monitoring application where the sensor network encompasses heterogeneous types of sensors distributed in different portions of a dam to gather data about the integrity of the dam. The sensor nodes are organized in multi-hop cluster based tree to sample the different physical parameters of the dam and route the reading to a central place for data management and visualization purpose. The application allows real-time collection and processing of sensor data presenting to the user through an appropriate interface (Seid, 2013).

\section{MATERIALS AND METHODS}

This study initially presents the main methods used to fulfill the DSM requirements. Furthermore, the building blocks of fuzzy inference systems also presented. As a result, a group of data on the input and output variables is gathered. The Fuzzy Inference System (FIS) variables are gathered and classified. The input variables to the water dam system consists of four fuzzification variables. The first one is the distance to the water of the dam specified by the ultrasound sensor of the system. The second variable is $\mathrm{X}$-axis reading of the accelerometer sensor of the system which measure the vibration in $\mathrm{X}$-axis of the dam body. The third variable is the $\mathrm{Y}$-axis reading of the accelerometer sensor of the system which measures the vibration in $\mathrm{Y}$-axis of the dam body. The last variable is the $Z$-axis reading of the accelerometer that measures the vibration in Z-axis of the dam body. These data modeled with the membership functions. The linguistic values of each variable are very low, low, medium, high and very high. Decisions have taken by fuzzy inference using fuzzy rules. The result is the amount of movement and direction of the dam gate depending on the dam condition determined by system sensors reading.

Water dam benchmark built and used to read the system sensors that incorporated in the dam control system. Real results studied to show how easy to use this methodology and how it it reflect the true behavior for dam monitoring. The paradigm of the control is if the water in the dam exceeds the normal level and the vibration sensors shows abnormal reading, the dam control system should adjust the dam gate, accordingly.

\section{System design and implementation}

Water dam benchmark monitoring techniques: The goals of safety monitoring of a dam is to classify as soon as possible, any irregularity which could be an symptom of some upcoming danger in order to allow adequate forewarning for the implementation of suitable corrective events (Urdiain et al., 2012). To accomplish these goalmouths, regular valuations of dam condition and behavior as well as periodic safety assessment are required. Regular assessment serves to monitor the current health of the dam, though periodic safety assessments serve to analyze long-term performance in order to detect abnormal tendencies. Generally, the supervision of the structural behavior confirmed by applying the following procedure:

- Acquisition of monitoring information, i.e., instrument measurements as well as qualitative information from visual inspections

- Interpretation and assessment of the information

- Taking decisions

- Archiving the results

The water dam instrumentation could be defined as the method and apparatus used to make physical capacities of dams. However, arrangement is not a supernumerary for review. It is a increment to the visual comments made during an examination. The requirements for instrumentation are more often precise to each individual dam site and require a large cost and man/ours. Some of these instrumentations comprise Piezometers, Inclinometers, Tiltmeters, Crackmeters and Jointmeters (Hu and Liu, 2011). These instrumentation-based methods conversely, require cabling which is luxurious. The cabling of a dam safety monitoring system is a convoluted project and the installation, adjustment and expansion of the monitoring points are very complex.

Water-dam monitoring system hardware: Water-Dam Monitoring System (WDMS) implemented on miniature water dam built for evaluation of the overall system work (Fig. 4). The WDMS prototype water dam made by wood and metal plates that can contain little amount of water. This mini water dam has a gate designed to work up and down by a small motor connected to the Arduino microprocessor. The model is also equipped with an ultrasonic sensor which measures the water height periodically and sends the result of the measurement to the microprocessor. A gyroscope-accelerometer sensor was also equipped to record deviations and vibrations in the body of the dam at three dimensions (X-Z). Figure 5 


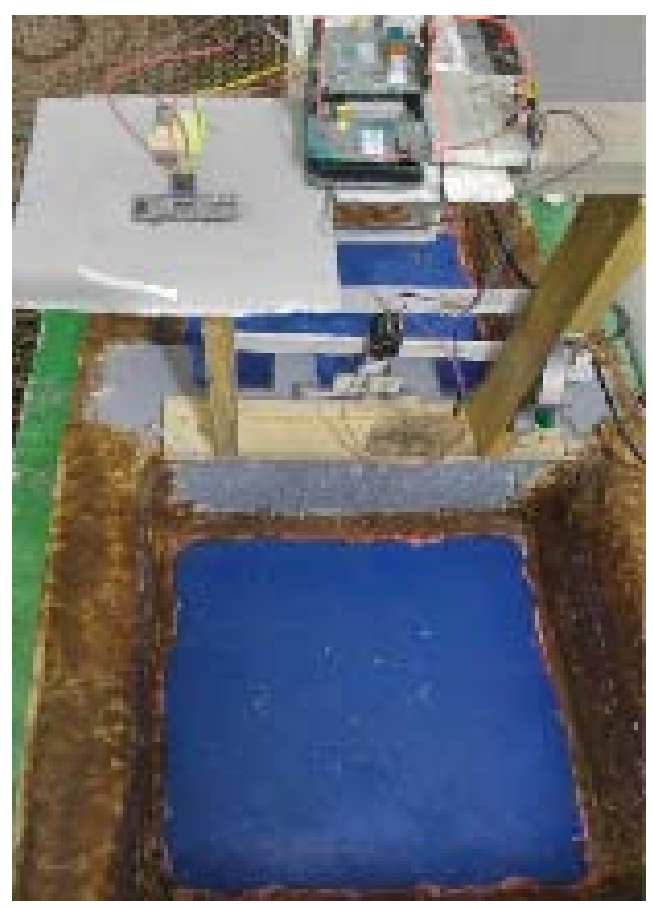

Fig. 4: Water dam miniature system

shows WDMS layout and organization. The WDMS workstation gather from the sink node the collected (X-Z) of Sensor Node Grid sensors (SND-Grid) using Zigbee protocol. These collected data processed using FIS controller that control the gate to be adapted with the state of dam.

WDMS architecture: Water-Dam Monitoring System (WDMS) implemented on miniature water dam built for evaluation of the overall system work. The WDMS prototype water dam made by wood and metal plates that can contain little amount of water. It used to evaluate the WDMS due to fact that real dam is difficult to maintain and a special sensors and loggers should be used. As shown in Fig. 4, the prototype consists of a dam foundation with proper water container supplied by the WDMS sensors and microcontroller. The dam gate designed using servomotor and the ultrasonic sensor installed above the gate and directed to the dam surface.

Sensor node: In this sensor, a set of sensors is deployed in the form of a matrix of sensors (SND-Grid) connected to a local network using the Zegbee protocol. These sensors simultaneously measure the vibration and deviation in the dam structure and the entire body of the dam. The sensor is mainly composed of an arduino microprocessor that reads the deviations and vibrations on the $\mathrm{X}$-axis (dam

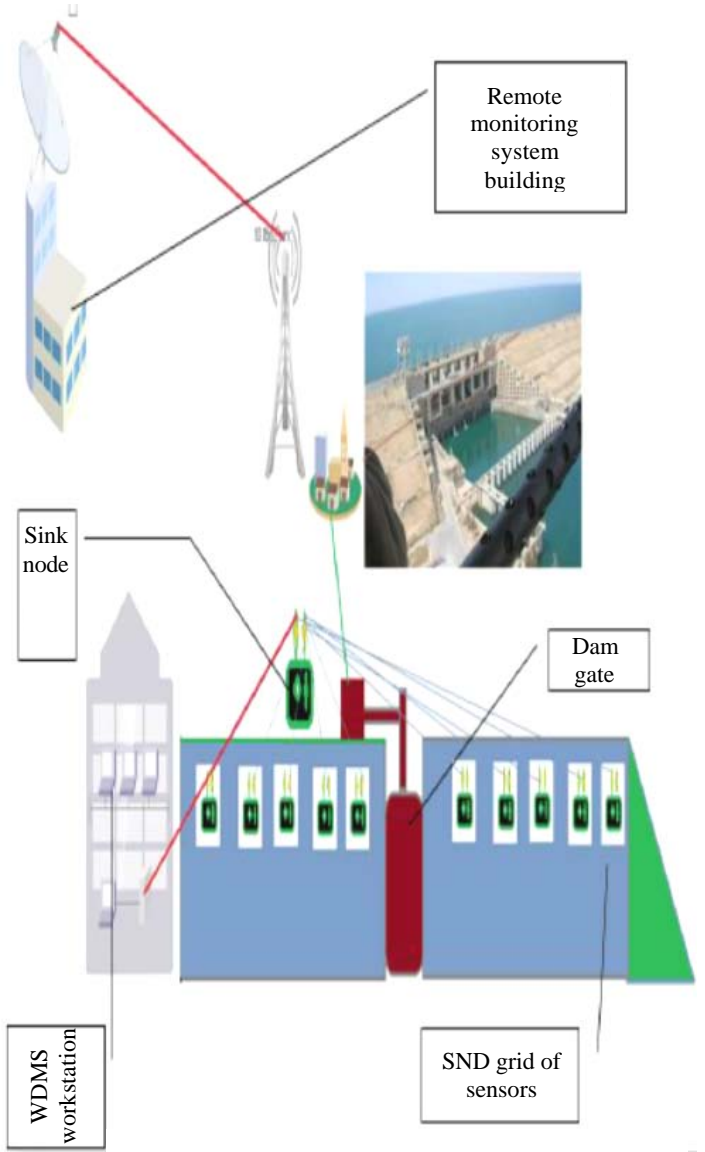

Fig. 5: Miniature water dam built evaluation of overall system work

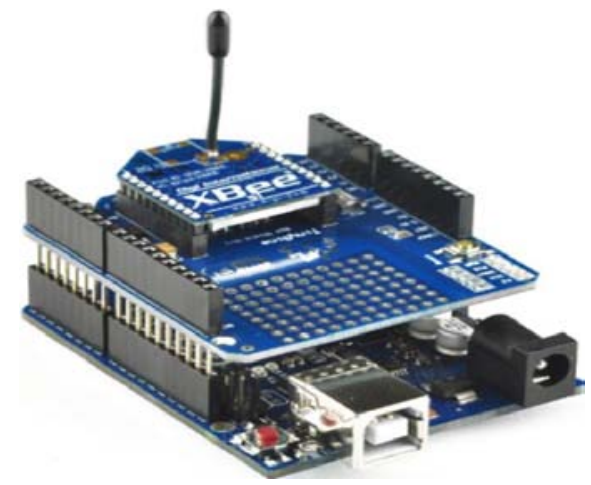

Fig. 6: Sensor node equipped with Xbee transceiver based on Zigbee protocol

extension) or the yaw (side-side) or the in-kind axis (the rise and fall of the dam structure) which causes the risk of cracking. The limits of oscillation to be compatible with the high level of water in the dam. This node consists of the following parts (Fig. 6). 


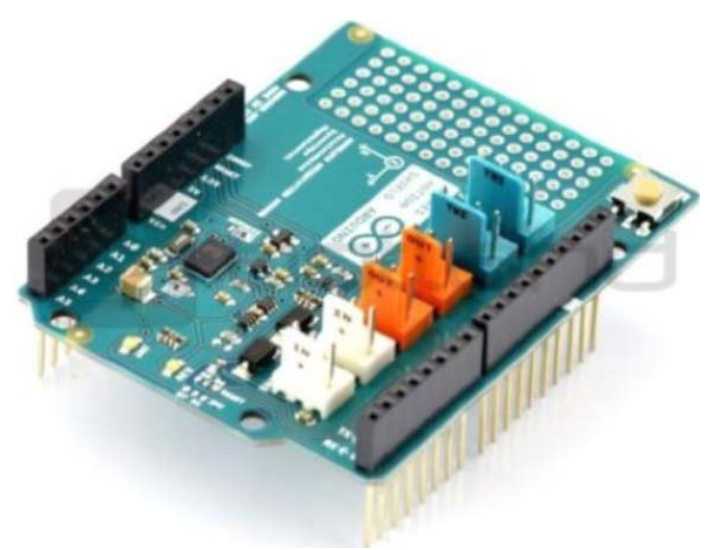

Fig. 7: Arduino 9-Axis Motion Shield outline

The Arduino Uno. is a microcontroller board based on the Atmega 328. It has 14 digital input/output pins (of which 6 can be used as PWM outputs), 6 analog inputs, a $16 \mathrm{MHz}$ ceramic resonator, a USB connection, a power jack, an ICSP header and a reset button. It contains everything needed to support the microcontroller; simply connect it to a computer with a USB cable or power it with an AC-to-DC adapter or battery to get started (Fig. 6).

Arduino Xbee shield: The Arduino Wireless shield allows the Arduino board to communicate wirelessly using Zigbee. It can be used as a serial/USB replacement or it can be placed into a command mode and configure it for a variety of broadcast and mesh networking options. The shields breaks out each of the Xbee's pins to a through-hole solder pad. It also provides female pin headers for use of digital pins 2-7 and the analog inputs which are covered by the shield (digital pins 8-13 are not obstructed by the shield, so, you can use the headers on the board itself). The Xbee shield has two jumpers. These determine how the Xbee's serial communication connects to the serial communication between the microcontroller and FTDI USB-to-serial chip on the Arduino board.

Arduino 9-Axes Motion Shield: The Arduino 9 Axes Motion Shield based on the BNO055 absolute sensor. The sensor features three-dimensional acceleration, yaw rate and magnetic field strength data each in 3 perpendicular axes. It also provides the sensor fusion signals such as (Fig. 7):

- Quaternion

- Euler angles

- Rotation vector

- Linear acceleration

- Gravity vector

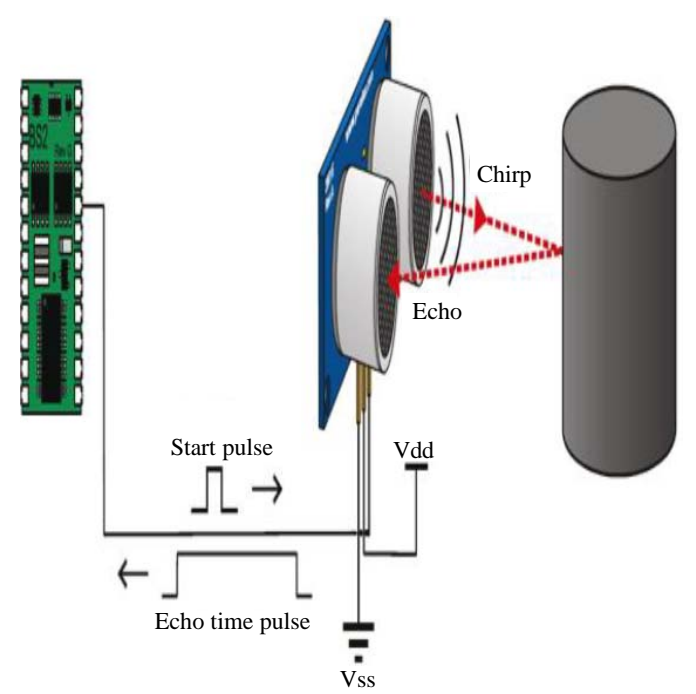

Fig. 8: Arduino ultrasonic distance sensor controller

Grid sensor uses this shield to capture acceleration in $\mathrm{x}$-z-axis, respectively. These environmental state of dam will be adjusted and transmitted to the Sink Node (SKN) to be used by embedded FIS algorithm for controlling the dam-gate.

Sink node: The Sink Node (SKN) receives the packets sent from the SND-grid sensors and forwarded to the main controller of this node (Arduino Mega) that burned with embedded FIS for adjusting and maintain the opening and closing dam-gate. This node also equipped with ultrasonic sensor that measure the distance of the water level of the water dam from the sensor.

Arduino Mega 2560: The Arduino Mega 2560 is a microcontroller board based on the ATmega2560. It has 54 digital input/output pins (of which 15 can be used as PWM outputs), 16 analog inputs, 4 UARTs (hardware serial ports), a $16 \mathrm{MHz}$ crystal oscillator, a USB connection, a power jack, an ICSP header and a reset button. It used for the node controller activities.

Arduino Xbee shild: Is the same as shield used in sink node (Fig. 8).

Arduino GSM shield: The primary use of the Arduino GSM shield is that it help to connect the Arduino or the GSM shield connects to the GPRS wireless network as a means of simple data packets. For this GPRS shield to work we just need to plug the GPRS module onto the Arduino borad, than after inserting a SIM card from a general mobile operator which offers data packet connection or the GPRS coverage in that area. 
Arduino ultrasonic distance sensor: This sensor is known as the ultrasonic distance senor. As the name implies it is a sensor which is used to determine the distance of an object from the sensor. Ultrasonic sensors work on a principle similar to sonar which evaluates attributes of a target by interpreting the echoes from sound waves, respectively. Ultrasonic sensors generate high frequency sound waves and evaluate the echo which is received back by the sensor. The time interval between the sent signal and received signal is determined to measure the distance from an object as indicated in Fig. 8.

Dam-gate motor: Servomotors are great devices that can turn to a specified position. Usually, they have a servo arm that can turn $180^{\circ}$. Using the Arduino, we can tell a servo to go to a specified position and it will go there. The main function of this motor in the SNK node is to lift up and down dam-gate with specified amount of movement determined by the SNK node controller.

\section{Water-dam monitoring system software}

Water-dam communication packet organization: For building an effective dam monitoring system, it is necessary to ensure that the dam works in conjunction with any emergency in the dam. The system monitors several factors that were addressed previously which include:

- The acceleration of the water dam body in X-axis

- The acceleration of the water dam body in Y-axis

- The acceleration of the water dam body in Z-axis

- The water level of the dam specified by the ultrasound sensor

The parameters are the most parameters chosen to reflect the dam state to the monitoring system. An ideal simple message block designed to ensure safe transfer of the dam state from the SN node to SKN node simultaneously and from the dam terminal to the remote central monitoring station. the architecture of the system message as follows:

\begin{tabular}{|l|c|c|c|c|c|c|c|c|c|}
\hline MO & val & LE & val & Ax & val & AY & val & AZ & val \\
\hline
\end{tabular}

Where:

Mo $=$ The message order that must be taken

$\mathrm{G} \quad=$ Get dam state

$\mathrm{S} \quad=$ Set dam state

$\mathrm{R}=$ Set the level to max and lift the damgate up to max

$\mathrm{M} \quad=$ Change the monitoring to manual state

$\mathrm{LE}=$ The water level in the dam has value (val) between $D-\min$ and max

$\mathrm{AX}, \mathrm{AY}, \mathrm{AZ})=$ The acceleration in $\mathrm{X}$-Z-axis of the dam

For example, the message:

\begin{tabular}{|l|l|l|l|l|l|l|l|l|l|}
\hline MO & $\mathrm{S}$ & $\mathrm{LE}$ & 10 & $\mathrm{AX}$ & 0 & $\mathrm{AY}$ & 0 & $\mathrm{AZ}$ & 0 \\
\hline
\end{tabular}

will set the D-gate to move to level 10 .

Sensor node grid acquisition algorithm: The process of measuring the deviation and acceleration of the dam cannot be accurate unless it measured along the body of the dam in order to make the right decision. Especially, that the great acceleration of the risk of cracking and rupture of the dam. A group of nodes is distributed along the body of the dam, so that, the dimension between the node and the others is constant. The 10 nodes were selected by default to calculate acceleration and deviation. The algorithm 1 shows the mechanism of gather SND-grid sensors reading.

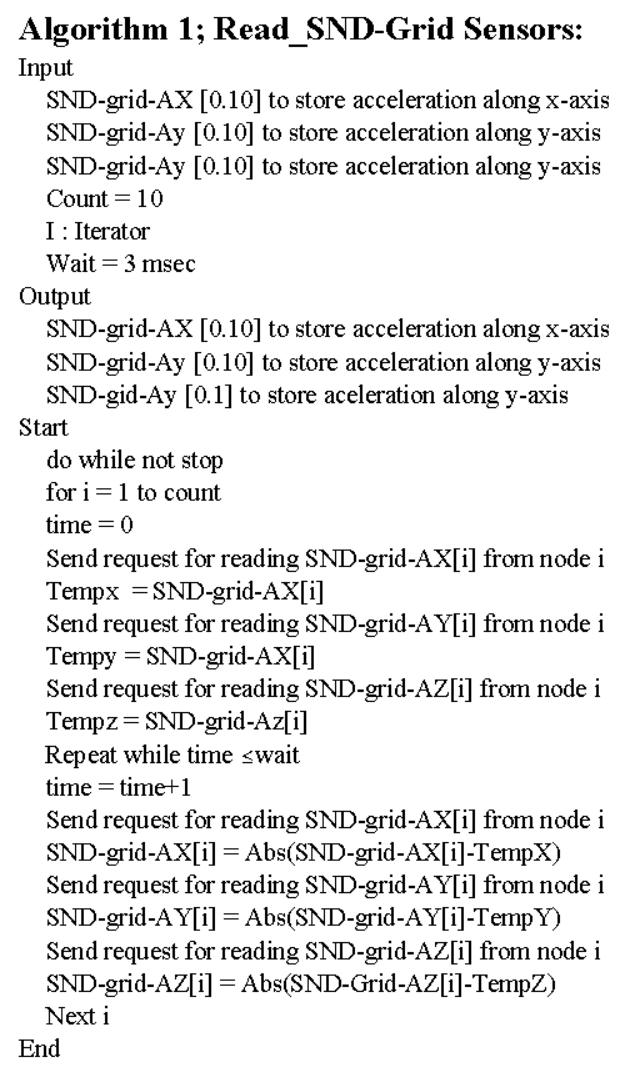


FIS controller: The FIS controller is the fuzzy controller resident in the sink node that receive all the message sent from the grid of sensors on the dam body that contain the information about the water level and the vibration information along the $\mathrm{XYZ}$ axis. FIS consists of four main parts: fuzzifier, rules, inference engine and defuzzifier. Our FIS has the following components.

Linguistic variables: The input or output variables of the system whose values are words or sentences from a natural language, instead of numerical values. A linguistic variable generally, decomposed into a set of linguistic terms. They are as follows:

- Input level denoted ad dam_level which has the linguistic \{too low, low, median, high too high\}

- Input accelerometer in X-axis denoted by Acc-X which has the linguistic \{too low, low, median, high, too high?

- Input accelerometer in Y-axis denoted by Acc-Y which has the linguistic \{too low, low, median, high, too high?

- Input accelerometer in Z-axis denoted by Acc-Z which has the linguistic \{too low, low, median, high, too high?

- Output dam-gate level denoted as gate-level which has the linguistic stoo low, low, median, high, too high?

Membership functions: There are different forms of membership functions such as triangular, trapezoidal, piecewise linear, Gaussian or singleton. The most common types of membership functions are triangular, trapezoidal and Gaussian shapes.

Fuzzy rules: In a FIS, a rule base is constructed to control the output variable. A fuzzy rule is a simple IF-THEN rule with a condition and a conclusion. Sample fuzzy rules for the dam monitoring system listed.

\section{Sample of WDMS fuzzy rule:}

- If (dam-level is too high or high) and (Acc-X is too high or high) then (gate-level is too high)

- If (dam-level is too high or high) and (Acc-Y is too high or high) then (gate-level is too high)

- If (dam-level is too high or high) and (Acc-Y is too high or high) then (gate-level is too high)

The FIS algorithm control the gate depending on the defuzzification value computed after executing the FIS on the embedded arduino controller in the sink node. FIS shown in Fig. 9.

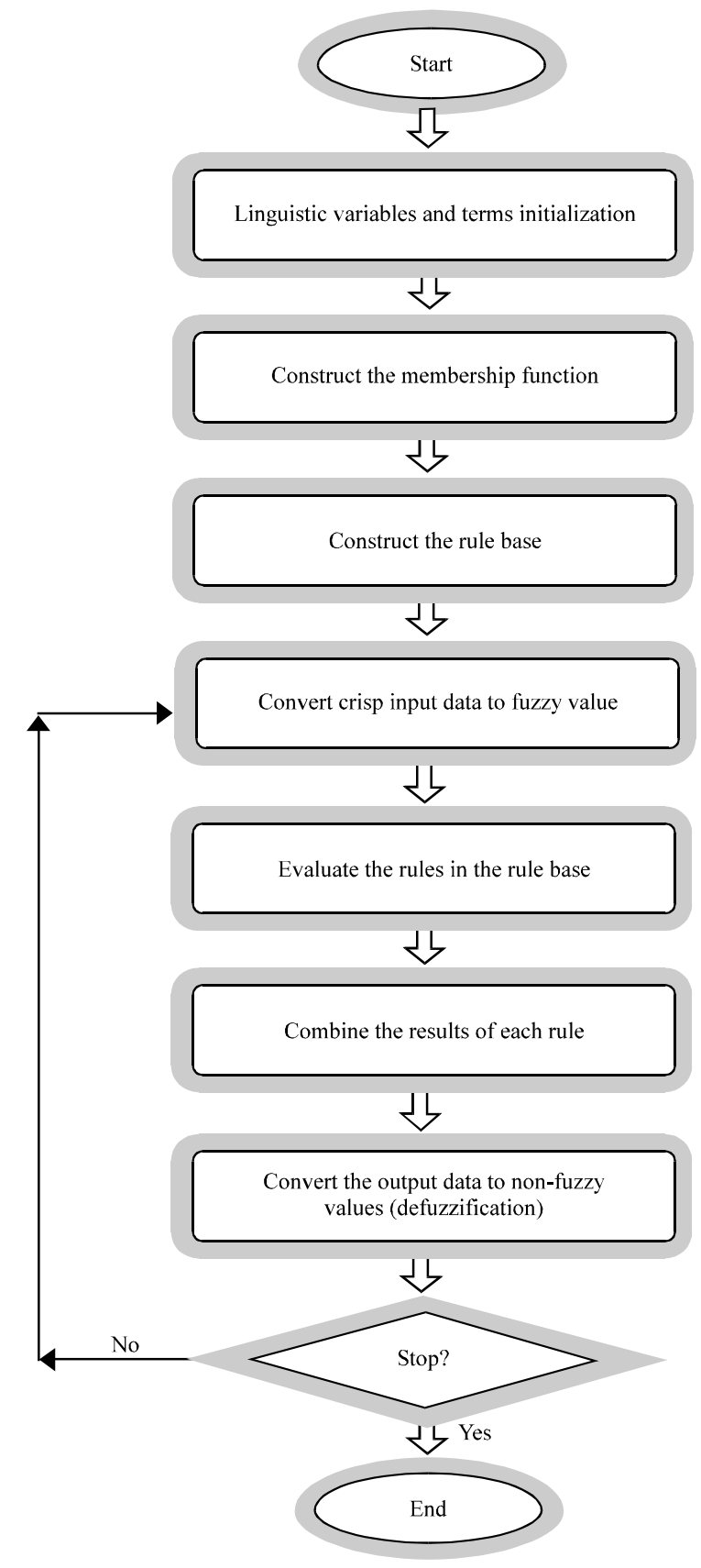

Fig. 9: Embedded fuzzy interface system

\section{RESULTS AND DISCUSSION}

WDMS consists of three main parts, the (SND-grid) collects the three dimensional vibrations from the dam body. Which sent to the (Dam Sink Node) DSN (the second part of WDMS) that the embedded FIS work to control the D-Gate autonomously depending on the (Dam State Matrix) DSM. These steps completely controlled 


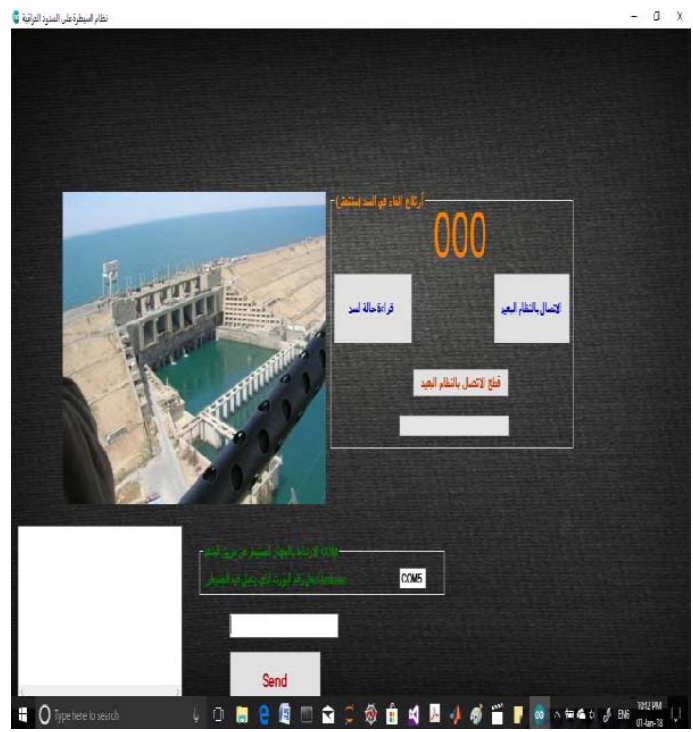

Fig. 10: Water dam monitoring system workstation

through the manual control from the WDMS workstation Dam-W(the third part). Dam-W also controlled remotely from the Remote Monitoring System (RMS) that implement simple interface to control WDMS system. The protocol adopted in this system built to maintain all these control messages.

Workstation system interface: Dam Workstation (D-W) part implemented using $\mathrm{C \#}$ for controlling the system. The system consists of simple interface as shown in Fig. 10. D-W attached to the DSN through serial communication link for testing. The attached DSN node supported and installed with the ultrasonic sensor for measuring the water dam level and the $10 \mathrm{WSN}$ nodes (SND-Grid) that connected to extract the (X-Z) vibration axis values. This system also used in the Remote Monitoring System (RMS) in this case, a GSM communication link used to control WDMS remotely. As seen from Fig. 10, the system used to test and monitor the system by sending appropriate commands directly to the DSN node. COM5 as serial port used to maintain direct serial connection.

Sink node fuzzy inference controller: The main part of any fuzzy system is how to derive the rule base from the fuzzy set depending on the desired behavior. WDMS rules where selected to ensure the proper opining and closing of W-gate. Since, the most important factor is the level of water in the dam, the rules derived concentrates on the dam level of water by assigning it the most important logic. Figure 11 shows WDMS fuzzy rules.

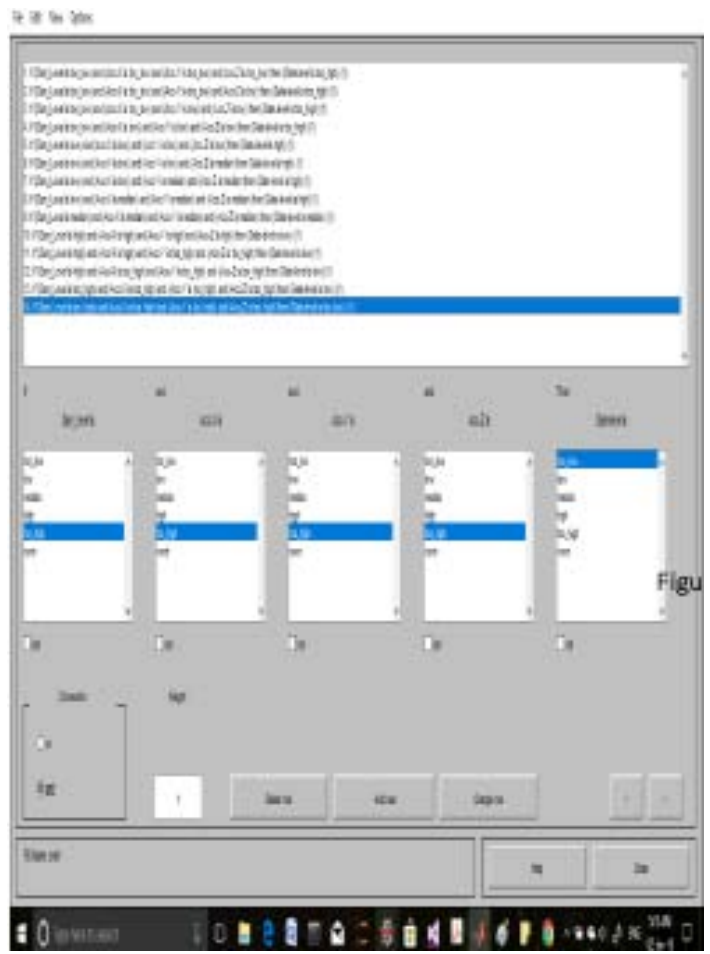

Fig. 11: WDMS fuzzy rule base

WDMS rules selected to ensure the proper opening and closing of $\mathrm{W}$-gate. Since, the most important factor is the level of water in the dam, the rules derived concentrates on the dam level of water by assigning it the most important logic.

Membership functions over linguistic variables: FIS linguistic variables are derived directly from the dam parameters that affected by dam operation. These parameters constructed as the reading of the dam sensors which include the WDMS fuzzy input (Dam-Level for the water level in the dam, Acc-X for accelerometer in $\mathrm{X}$-axis, Acc- $\mathrm{Y}$ for accelerometer in $\mathrm{Y}$-axis and Acc-Y for accelerometer in $Z$-axis). The fuzzy output represented by one parameter (Gate-Level) which indicates the level of the dam gate that the motor will drive. Figure 12 shows the membership functions of the linguistic variable for $\mathrm{X}$-axis.

After constructing linguistic variables, rule base system and the membership functions, the FIS configured and ready for evaluation. The membership function used in the system is triangular-shaped membership function. Figure 13 shows the FIS used for WDMS.

FIS controller evaluation: FIS play the central part in the WDMS for smoothly control the dam gate and adjust its 
operation adaptively with dynamics of the dam parameters. Gaussian curve membership function was used in comparison against Triangular-shaped membership function. The Gaussian curve membership function defined as:

$$
f(x ; \sigma, c)=e^{\frac{-(x-c)^{2}}{2 \sigma^{2}}}
$$

Where:

$\mathrm{X}=$ The data vector

$\sigma=$ The variance

$\mathrm{C}=$ Constant

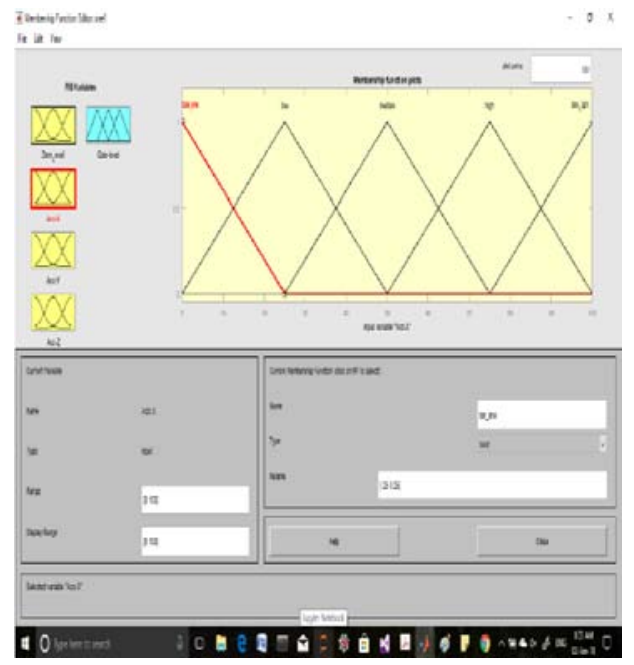

Fig. 12: Membership function of the dam-level fuzzy variable
Triangular-shaped membership function: We generate test input data using random number generator for 100 reading of simulated dam parameters. FIS controller was executed and the results was indicated in Fig. 14.

Gaussian curve membership function: Same data above were used tom evaluate linguistics using Gaussian curve membership function. The results indicated in Fig. 15. Surface evaluation also tested for linguistic variables as shown in Fig. 16 and 17. Arduino editor used to program and flush the microcontroller Mega and Uno.

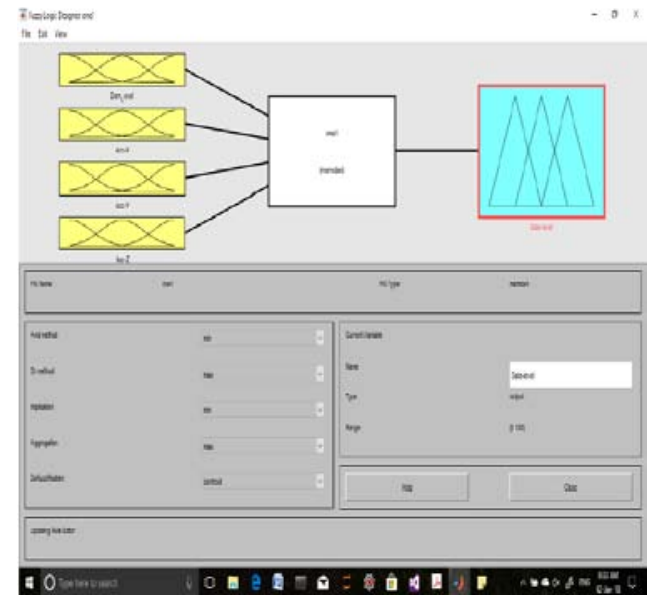

Fig. 13: WDMS fuzzy controller system

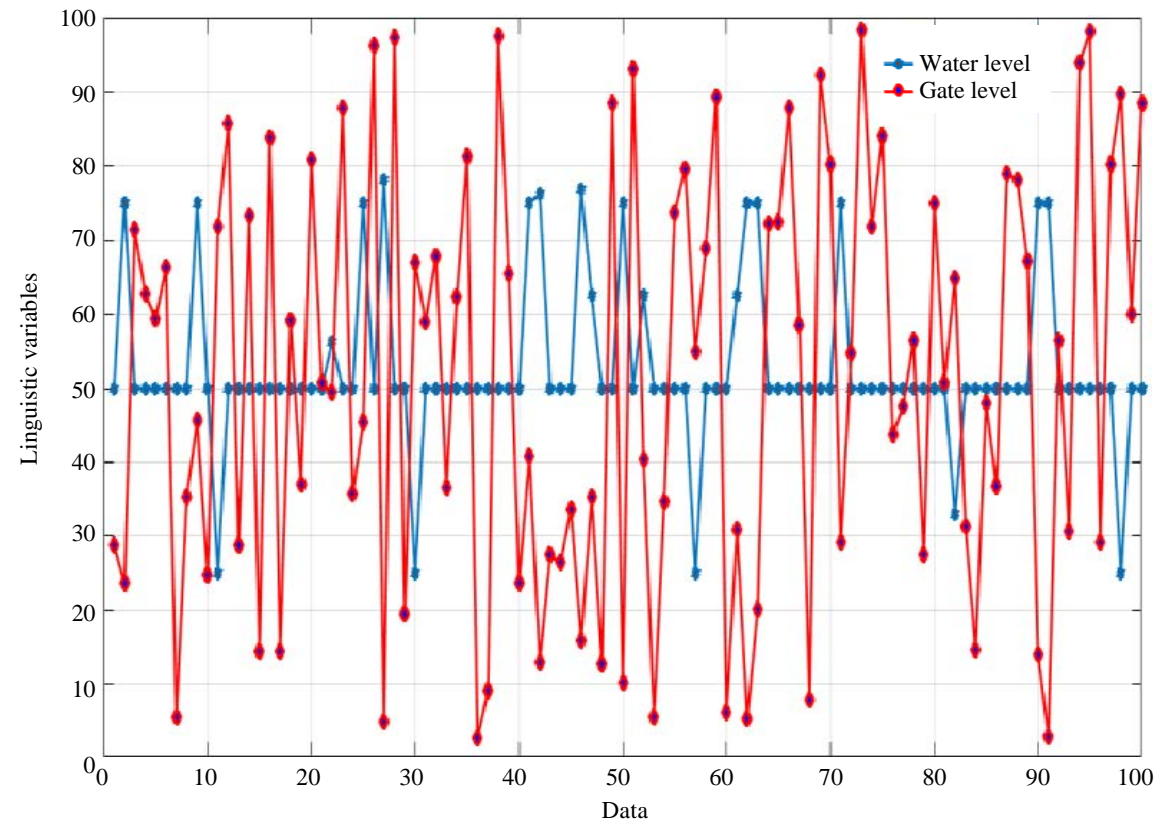

Fig. 14: Water level versus gate level with triangular membership 


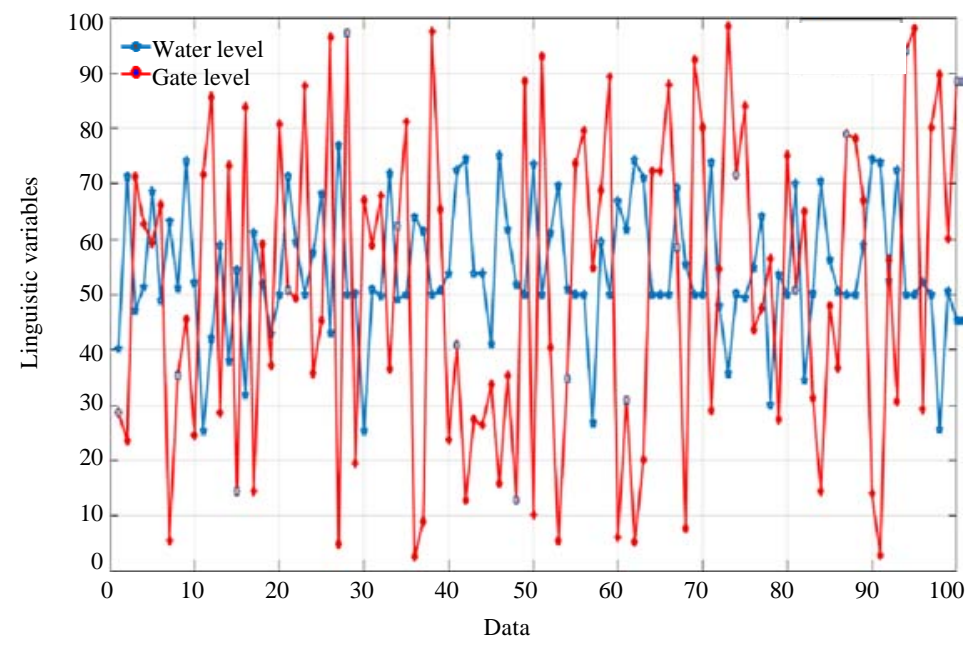

Fig. 15: Water level versus gate level with Gaussian membership

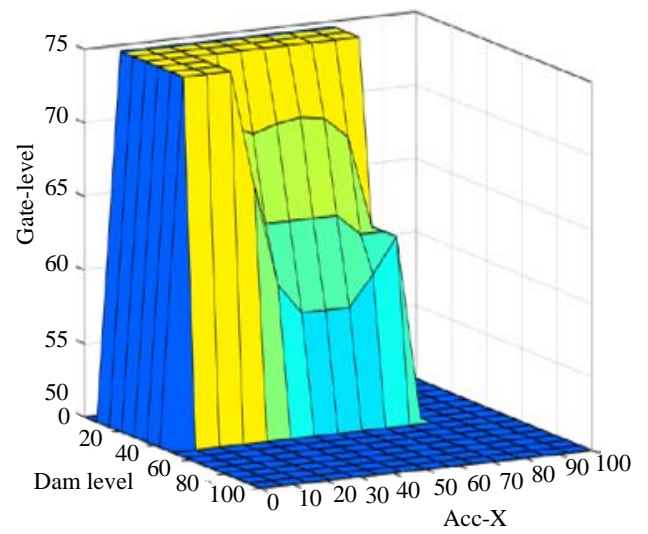

Fig. 16: Surface view of linguistic variable using triangular membership

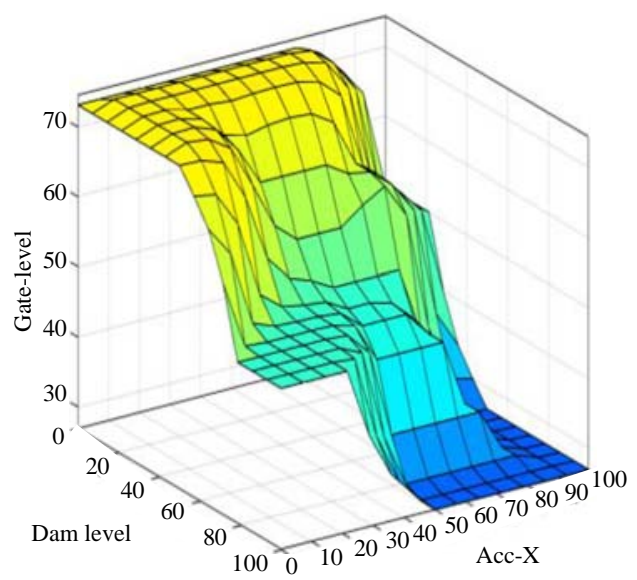

Fig. 17: Water level versus gate level with Gaussian membership

\section{CONCLUSION}

In this study, it was shown that the water dam miniature benchmark worked successfully within the proper response to the different water filling policies. The FIS controller in these Sink node shows good adaptation to the changing in the water level and the vibration applied to the dam. The proposed system prototype was successfully mimic the real water dam in case of reading the vibration waves and existing of controlled gate. The GSM communication protocol was superior than LAN/WAN based communication protocol in case of availability and cost. The node sensor configuration along the dam body ensure consistence reading as it form the WSN that adapt quickly and efficiently. In addition, FIS controller was able to smoothly control the gate and prevent the hazard of dam flood fill. Form the workflow of the system, the Gaussian membership was more efficient than triangular membership function and adapt more quickly.

\section{REFERENCES}

Hu, J. and X. Liu, 2011. Design and implementation of tailings dam security monitoring system. Procedia Eng., 26: 1914-1921.

Imran, M., M. Zulfqar, H. Rasheed, S. Tayyaba and M.W. Ashraf et al., 2016. Fuzzy logic based flow controller of dam gates. J. Eng. Res. Technol., 1: 83-90.

Kavanagh, M., 2011. Modes of Dam Failure and Monitoring and Measuring Techniques. Environment Agency, Bristol, UK., ISBN: 978-1-84911-230-7, Pages: 162. 
Koubaa, A., A. Mario, M. Attia and A. van Nieuwenhuyse, 2006. Collision-free beacon scheduling mechanisms for IEEE 802.15.4 Zigbee cluster-tree wireless sensor networks. J. Comput. Sci., $1: 1-8$.

Manes, G., R. Fantacci, F. Chiti, M. Ciabatti and G. Collodi et al., 2007. Enhanced system design solutions for wireless sensor networks applied to distributed environmental monitoring. Proceedings of the IEEE 32nd International Conference on Local Computer Networks (LCN 2007), October 15-18, 2007, IEEE, Dublin, Ireland, pp: 807-814.

Miao, X., J. Chu, L. Zhang and J. Qiao, 2012. Development of wireless sensor network for dam monitoring. J. Inf. Comput. Sci., 9: 1609-1616.

Nanaware, R.A., S.R. Sawant and B.T. Jadhav, 2012. Fuzzy based decision system for gate limiter of hydro power plant. Intl. J. Electron. Electr. Eng., 5: 157-166.

Pan, M.S. and T. Yu-Chee, 2006. Zigbee wireless sensor networks and their applications. MSc Thesis, National Chiao Tung University, Hsin-Chu, Taiwan.

Sazonov, E., K. Janoyan and R. Jha, 2004. Wireless intelligent sensor network for autonomous structural health monitoring. Proceedings of the International Conferences on Smart Structures and Materials 2004: Smart Sensor Technology and Measurement Systems Vol. 5384, July 27, 2004, SPIE, Washington, USA., pp: 305-315.

Schurer, J., E. Wilkinson, J. Norfleet, J.W. van Sciver and C. Huntington et al., 2002. Dam Safety Manual. State Engineer's Office State of Colorado, New York, USA., Pages: 141.
Seid, S., 2013. Dam safety monitoring using wireless sensor networks. Ph.D Thesis, Addis Ababa University, Addis Ababa, Ethiopia.

Shukla, S. and G. Pandey, 2014. To design an architectural model for flood monitoring using wireless sensor network system. Intl. J. Comput. Sci. Inf. Technol., 5: 502-507.

Sohraby, K., D. Minoli and T. Znati, 2007. Wireless Sensor Networks: Technology, Protocols and Applications. John Wiley \& Sons, Hoboken, New Jersey, USA., ISBM:978-0-471-74300-2, Pages: 307.

Telgote, A.M., V. Narayanan and N.A.N. Dave, 2015. Design and implementation of water environment monitoring system using GSM technology. Proceedings of the International Conference on Technologies for Sustainable Development (ICTSD), Febraury 4-6, 2015, IEEE, Mumbai, India, ISBN: 978-1-4799-8186-1, pp: 1-4.

Urdiain, L.O., C.P. Romero, J. Doggen, T. Dams and P. Van Houtven, 2012. Wireless sensor network protocol for smart parking application experimental study on the Arduino platform. Proceedings of the 2nd International Conference on Ambient Computing, Applications, Services and Technologies (AMBIENT 2012), September 23-28, 2012, Ambient Light Publishers, Barcelona, Spain, ISBN: 978-1-61 208-235-6, pp: 45-48.

Wagh, T.R. and A.P. Rao, 2014. River monitoring an application for wireless sensor network platform. Intl. J. Emerging Technol. Adv. Eng., 4: 511-515. 